\title{
Quantum Structure of Geometry: Loopy And FuZzy?
}

\author{
Alejandro Corichi ${ }^{1,2, *}$ And José A. Zapata ${ }^{1,+}$ \\ ${ }^{1}$ Instituto de Matemáticas - Morelia, \\ Universidad Nacional Autónoma de México, \\ UNAM-Campus Morelia, A. Postal 61-3, \\ Morelia, Michoacán 58090, Mexico. \\ ${ }^{2}$ Institute for Gravitational Physics and Geometry, Physics Department, \\ Pennsylvania State University, University Park PA 16802, USA
}

\begin{abstract}
In any attempt to build a quantum theory of gravity, a central issue is to unravel the structure of space-time at the smallest scale. Of particular relevance is the possible definition of coordinate functions within the theory and the study of their algebraic properties, such as non-commutativity. Here we approach this issue from the perspective of loop quantum gravity and the picture of quantum geometry that the formalism offers. In particular, as we argue here, this emerging picture has two main elements: i) The nature of the quantum geometry at Planck scale is one-dimensional, polymeric with quantized geometrical quantities and; ii) Appropriately defined operators corresponding to coordinates by means of intrinsic, relational, constructions become non-commuting. This particular feature of the operators, that operationally localize points on space, gives rise to an emerging geometry that is also, in a precise sense, fuzzy.
\end{abstract}

PACS numbers: $04.60 . P p$

*Electronic address: corichi@matmor.unam.mx

$\dagger$ Electronic address: zapata@matmor.unam.mx 
The question of what the microstructure of space-time is has been in the minds of many theoretical physicists for the past 80 years (if not more), ever since it was clear to Einstein in 1916 that we need a grander theory that unites gravity and the quantum: a quantum theory of gravity. We do not yet have such a satisfactory framework that brings together the quantum and gravitational realms. However, there are questions that can be posed even in the absence of a final theory. For instance, one can ask whether there is a minimal length scale in the theory (See [1] for a review). Another question that comes about is the issue of the structure of space-time at this scale. The nature of this question is however, a bit less precise since the question itself depends heavily on the details of the approach one is considering. For instance, if one assumes the existence of space-time (as a 4-dimensional background manifold) as a-priory given, one will pose different questions as one would in purely canonical approaches where the theory is defined on a 3-manifold $\Sigma$ and where a 4-dimensional space-time emerges only in some semi-classical regime.

One particular feature of the underlying structure of quantum space-time is a possible noncommutativity at the fundamental scale. Somewhat surprisingly, even in this case, there seems to be no consensus regarding what this means exactly. Sometimes it is assumed that the coordinates will be non-commutative while, alternatively, non-commutativity could be understood in the sense of Connes' non-commutative geometry [2], where not even the notion of a manifold survives. More recently, a pile of results coming from different approaches hint to a possible non-commutative structure at the fundamental scale of the theory. This has been extensively explored in the literature on string theory, gauge fields and in other frameworks, so we will not review it here. Some of these approaches suffer, we believe, from a serious drawback: While their objective is to understand the micro structure of space-time in the attempt to go down to the most basic object on which physics is constructed, their starting point is not a fundamental object. General relativity taught us more than 90 years ago that coordinates are not fundamental objects for the description of the physics, and any approach that uses them as a starting point would seem to contradict the lessons of GR. Once the dynamical objects, described in terms of fields (gravitational included) have been prescribed, then one could construct a notion of coordinates defined dynamically from the theory itself (such as the so-called GPS coordinates [3]). Taking this lesson seriously implies that any notion of non-commutative coordinates as a starting point faces the burden 
of proving itself consistent with background independence.

In this note we shall advocate a different perspective on the structure of spacetime; a picture that has arisen from the framework known as loop quantum gravity (LQG) [4]. To begin with, as presently understood, loop quantum gravity is a theory defined on a canonical setting, which means that there is no 4-dimensional object we can call space-time, but only a background 3-manifold $\Sigma$ where the theory is defined. Space-time becomes a derived object. There is no background metric $q_{a b}^{\mathrm{o}}$ defined on $\Sigma$, so the quantum theory has to be defined, in this sense, metric background-independent. What makes LQG radically different from other canonical approaches to quantum gravity (such as its predecessor, Quantum Geometrodynamics in terms of the Wheeler-DeWitt equation), is that it can actually be well defined. More precisely, we have for the first time a mathematically consistent framework where some of the basic objects, such as a Hilbert space and finite operators, exist (For details see [4] and for pedagogical introductions see [5] and [6]). A crucial input in the construction is the choice of objects that are rendered as basic for the quantization of the theory, namely connections and loops. Connections are present because Einstein's theory was recast as a gauge theory with a Yang-Mills structure; loops are important because they are the natural objects to use when defining simple functions of the connection, namely, parallel transports and holonomies. The metric information on $\Sigma$ is contained in the triads $e_{i}^{a}$ that are the other objects containing the full information of the phase space. Any 3-geometric object is constructed out of this canonical variable.

An important aspect of the formalism is that, apart from the choice of variables, one does not presuppose the nature of the microstructure of space nor its properties. There is no input about discreteness at any scale. One literally lets the theory lead.

From these basic objects, one can construct quantum operators that represent geometrical quantities such as areas of surfaces $\hat{A}[S]$ and volumes of regions $\hat{V}[R]$. The operators are finite and suffer from no regularization infinities. They can be rigourously regulated without the need of any renormalization. With them one can explore the nature of the quantum geometry that Loop Quantum Gravity offers for us. The first thing that the formalism tells us is that the quantum (metric) geometry is concentrated on lower dimensional objects. Loops (or more generally, edges of graphs) carry "fluxes of area", very much in the style of 
Faraday, while vertices of the graph are responsible for the volume of regions. The second important feature is that the spectra of the operators are discrete, providing in a precise sense a quantization of the geometry. Thus, if $\left|\Psi_{\Upsilon, \vec{j}}\right\rangle$ is a quantum state labelled by a graph $\Upsilon \in \Sigma$ and a set of labels $\vec{j}$, one for each edge, then the action of the operator $\hat{A}[S]$ on $\left|\Psi_{(\Upsilon, \vec{j})}\right\rangle$ is given by,

$$
\hat{A}[S] \cdot\left|\Psi_{(\Upsilon, \vec{j})}\right\rangle=\left(8 \pi \gamma \ell_{\mathrm{pl}}^{2} \sum_{v_{i}} \sqrt{j_{i}\left(j_{i}+1\right)}\right)\left|\Psi_{(\Upsilon, \vec{j})}\right\rangle,
$$

where the sum is over all the intersection points $v_{i}$ between $S$ and $\Upsilon$, and the (half integer) $j_{i}$ corresponds to the label of the corresponding edge. In particular, note that there is a minimum area gap in the theory corresponding to a single intersection and spin $j=1 / 2$ : $A_{\text {min }}=4 \pi \gamma \ell_{\mathrm{pl}}^{2} \sqrt{3}$. The real number $\gamma>0$ is a free parameter of the theory that needs to be fixed by some 'experiment' (it is the analogue of the $\theta$ parameter in QCD). The best (at present) proposal for fixing the value of the parameter comes from Black Hole physics and it is of the order of $\gamma=0.274 \ldots$. With this choice the minimum area gap $A_{0} \approx 6 \ell_{\mathrm{pl}}^{2}$ is of the order of the Planck area. This is the smallest area possible and as we have already emphasized, it is a prediction of the theory.

The third, and somewhat unexpected feature, is that the loop quantum geometry is inherently non-commutative: operators associated to intersecting surfaces $S_{1}$ and $S_{2}$ do not commute:

$$
\left[\hat{A}\left[S_{1}\right], \hat{A}\left[S_{2}\right]\right] \neq 0 .
$$

For more details see [7, 8]. This, of course implies that there is an intrinsic uncertainty in the geometry, and therefore an induced fuzziness in the spatial quantum geometry. Any attempt of localization by means of operators associated to surfaces will be subject to a basic Heisenberg uncertainty $\left.\left(\Delta A\left[S_{1}\right]\right)\left(\Delta A\left[S_{2}\right]\right)\right) \neq 0$. Many questions come to mind regarding the non-commutativity of these fundamental geometric operators. In particular, one may wonder how we can recover an apparently smooth, commutative geometry on large scales? How does this affect the algebraic nature of symmetry groups, such as the Lorentz group, that are so useful in describing physics at low energies? Does this non-commutativity survive in the full dynamical setting?, etc.

So far, we only have partial answers to some of these questions, but the picture of the 
geometry of space that LQG presents for us is quite novel and intriguing. Let us now explore some of these new aspects of this quantum description of geometry at the fundamental scale. For instance one question that is basic to any further consideration is the following: In what sense can we regard individual points $p_{j}$ of the manifold $\Sigma$ as endowed with a physical meaning? In other words, does the concept of a point on space even make sense? In the context of classical general relativity we know the answer: given that the theory is invariant under diffeomorphisms, the concept of a point as an abstract entity dissolves. Instead what makes sense is the point not as an abstract mathematical object but as the location where matter fields and gravity have some particular property (for instance the point where two word-lines intersect, or the point where light is emitted by a source, etc.). Nevertheless, any point on the manifold is as good as any other point given that all fields are smooth objects and are thus 'well defined' on any point of space. What is then the situation in the quantum realm? After all, quantum fields are a very different class of objects than its classical counterparts.

Is loop quantum geometry point-less? Let us start to answer that question by considering

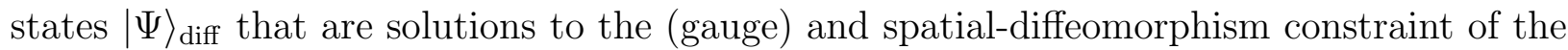
theory. In terms of the objects $\left|\Psi_{\Upsilon, \vec{j}}\right\rangle$, they can be obtained by means of an average over the orbits of the diffeomorphisms acting on the state. In a sense the original graph $\Upsilon$ is no longer localized on the manifold, but is 'smeared out by means of the average' procedure. What matters of the state $|\Psi\rangle_{\text {diff }}$ are the self relational properties of the original state $\left|\Psi_{\Upsilon, \vec{j}}\right\rangle$. That is, the incidence relations of the graph $\Upsilon$, the labels $j_{i}$ on the edges, etc. It should be clear then that it is only the graph and points on it that can be given any physical meaning. There are simply no points 'outside the graph and on the manifold $\Sigma$ '. The manifold $\Sigma$ itself has dissolved. The next observation is that within a graph (that can be seen as abstract) there is a clear distinction between points on it. Thus, points that lie on an edge are qualitatively different from those that are vertices (with more than two incident edges). In terms of the geometric operators with which we can probe the state (and thus determine what is the quantum geometry defined by the state), a point $p_{e}$ on an edge can carry a flux of area (detected by acting on the state with a surface intersecting the graph $\Upsilon$ at the point $p_{e}$ ), but it corresponds to vertices $p_{v}$ on $\Upsilon$ the privilege of endowing 3-dimensional regions $R$ with a volume; the corresponding volume operator only 'excites' vertices with valence four 
and higher. Otherwise, the contribution to the volume is zero. Furthermore, when coupling any kind of matter, it is again the vertices with volume the ones that contribute by providing the volume for the matter Hamiltonian. Thus, if one is to define the meaning of "points of space' in a relational manner, by means of dynamical matter fields, it will only be vertices of the graph that will be elevated to the level of physical points.

Having established this fact, one could try to define the notion of coordinates of physical points and study their properties. For instance, if we define in a relational manner, using only objects defined on the graph and geometrical quantities, operators $\hat{Q}_{i}$ assigning coordinates to physical points, it turns out that the resulting operators are non-commuting. Let us now explain how one can define this type of operators (the choice is by no means unique). The underlying principle behind these constructions is that the coordinates have to be defined intrinsically from the dynamical objects of the theory. In this sense they become relational.

Let us first consider the situation for the definition of these coordinate functions in the classical scenario. Our construction will use a fiducial metric $q_{o}$ on $\Sigma$, and a set of reference points $\left(p_{0}, p_{1}, p_{2}, p_{3}\right)$ to construct the coordinate functions (functions on phase space). We will use the fiducial metric and the reference points to construct, given any point $p$ 'near $p_{i}{ }^{\prime}$, three 2 -spheres $S_{i}$ as follows: The sphere $S_{1}$ for example will be the $q_{0}$-sphere that has $\left(p_{0}, p_{2}, p_{3}, p\right)$ on it. That is, it is the (unique, provided some tame conditions are satisfied) sphere defined by these four points. In the same manner, we can define $S_{2}$ by the four points $\left(p_{0}, p_{1}, p_{3}, p\right)$, and $S_{3}$ by $\left(p_{0}, p_{1}, p_{2}, p\right)$. Now we define the coordinates,

$$
Q_{i}:=A\left[S_{i}\right]
$$

That is, the coordinates are given by the value of the area corresponding to the $S_{i}$ sphere, as given by the metric on $\Sigma$ defined by the phase space point (not the fiducial metric). In a neighborhood of the reference points, we are able to define the spheres and thus the coordinates $Q_{i}$. As in the definition of any coordinate chart, the choices of the reference points and the fiducial metric are totally arbitrary. Any other choice will, of course give different coordinates for the same point. We again remark that these coordinates are ' $q$ coordinates' in the sense they depend on the phase space point (in this case, the spatial metric). 
Let us now try to export the construction to the quantum realm. First we have to specify the reference points $\left(p_{0}, p_{i}\right)$. From the discussion before we can only choose vertices on the graph $\Upsilon$ (physical points) as the reference points and furthermore, the only points $p$ for which the coordinates will be defined are also physical points. In the construction we will make use of the fiducial metric to select the surfaces $S_{i}$; as we have mentioned for the classical coordinates, this is part of the arbitrary specification of any coordinate system. The second observation is that we shall define the corresponding operators in the kinematical setting, where the graphs are embedded into $\Sigma$. The objects to be 'quantized' are the area functions that now will be replaced by area operators as,

$$
\hat{Q}_{i}:=\hat{A}\left[S_{i}\right]
$$

The important thing to note is that the point $p$ corresponds to the point where all the spheres $S_{i}$ intersect, and given that $p$ is a physical point, it is a node with al least valence four in the graph. This is precisely the condition for the area operators not to commute. Thus, in general, we have that,

$$
\left[\hat{Q}_{i}, \hat{Q}_{j}\right] \neq 0
$$

The coordinate operators associated to physical points are non-commuting. Needless to say, we have employed a rather simple prescription, so our results are not exhaustive. In the small sample of 'slightly more elaborate constructions' that we have explored, we have also found that the corresponding quantum coordinates do not commute [8].

In a situation where matter fields have been coupled to loop quantum gravity, we could define similar coordinate operators whose basic building blocks are the area operators $\hat{A}\left[S_{i}\right]$, but this time the surfaces $S_{i}$ would be specified using dynamical fields. It is exactly this situation what we had in mind when we demanded that the point to be assigned coordinates and the reference points had to be physical points (nodes of the graph that specifies the state). These coordinate operators would be background independent and they would still not commute.

The construction so far was somewhat kinematical since we employed a particular fiducial metric and an embedding of the graph into the manifold $\Sigma$. Can we elevate our construction to the diffeo invariant realm? That is, without the need for a particular embedding? The answer is in the affirmative but the construction is somewhat subtle. In our construction of coordinate operators we used both the fiducial metric and the embedded graph that defines 
the $q$-arena we are considering: the quantum geometry defined by the graph $\Upsilon$ and the state $\Psi_{\Upsilon}$ defined on it. The fiducial metric allowed us to construct the auxiliary quantities such as the spheres defined by the reference points and the point to be measured. But once this objects are specified and the operators $\hat{Q}_{i}$ are defined, they only know about 'combinatorial' aspects of the state: the vertex on which they act and the particular action on the incoming edges to that vertex. We can extend the action of these operators to states obtained by the action of a diffeomorphism $\phi$ : simply maintain the same action on the 'internal' part of the state, for all states on the orbit of the diffeomorphism group. Defined in such a way, the operators become well defined on the space of diffeo-invariant states. This procedure can be seen as the equivalent, on the quantum realm, of the "evolving constants of the motion" prescription on the classical side where a gauge invariant function is defined as constant along the orbits of the constraint functions.

The scenario here presented is by no means conclusive, but certainly these results hint to a geometry at the Planck scale that is not only polymeric, but also intrinsically noncommutative. This represents the first example of concrete non-commutativity arising as a consequence of a well defined mathematical framework that unites the gravitational and quantum realms. ${ }^{1}$ Needless to say, the story is far from over and the subject deserves further attention. We hope to report the results of such investigations in a near future [8].

Apart from the possible non-commutativity of space-time, and just as general relativity changed so drastically our notions of space and time, a final theory of quantum geometry will for sure bring a new and deeper perspective to these notions. What we can all probably agree upon is that is are still far from a definite answer.

We thank J.M. Reyes for discussions. This work was partially supported by CONACyT

\footnotetext{
${ }^{1}$ Perhaps the most notable exception are the intriguing results by Freidel and collaborators [9]
} 
U47857-F and NSF PHY04-56913 grants, by the Eberly Research Funds of Penn State.

[1] L. J. Garay, "Quantum gravity and minimum length," Int. J. Mod. Phys. A 10 (1995) 145 arXiv:gr-qc/9403008.

[2] A. Connes, "Noncommutative differential geometry and the structure of space time," Prepared for NATO Advanced Study Institute on Quantum Fields and Quantum Space Time, Cargese, France, 22 Jul - 3 Aug 1996

[3] C. Rovelli, "GPS observables in general relativity," Phys. Rev. D 65, 044017 (2002) arXiv:gr-qc/0110003.

[4] A. Ashtekar and J. Lewandowski "Background independent quantum gravity: A status report," Class. Quant. Grav. 21 (2004) R53 arXiv:gr-qc/0404018]; C. Rovelli, "Quantum Gravity", (Cambridge U. Press, 2004); T. Thiemann, "Introduction to modern canonical quantum general relativity," arXiv:gr-qc/0110034.

[5] A. Ashtekar, "Gravity and the quantum," New J. Phys. 7 (2005) 198 arXiv:gr-qc/0410054.

[6] A. Corichi, "Loop quantum geometry: A primer," J. Phys. Conf. Ser. 24 (2005) 1 arXiv:gr-qc/0507038.

[7] A. Ashtekar, A. Corichi and J. A. Zapata, "Quantum theory of geometry. III: Non-commutativity of Riemannian structures", Class. Quant. Grav. 15 (1998) 2955 arXiv:gr-qc/9806041];

[8] A. Corichi, J.M. Reyes and J.A. Zapata, "On the non-commutative geometry of loop quantum gravity" Preprint

[9] L. Freidel and E. R. Livine, "Effective 3d quantum gravity and non-commutative quantum field theory," Phys. Rev. Lett. 96, 221301 (2006) arXiv:hep-th/0512113. 